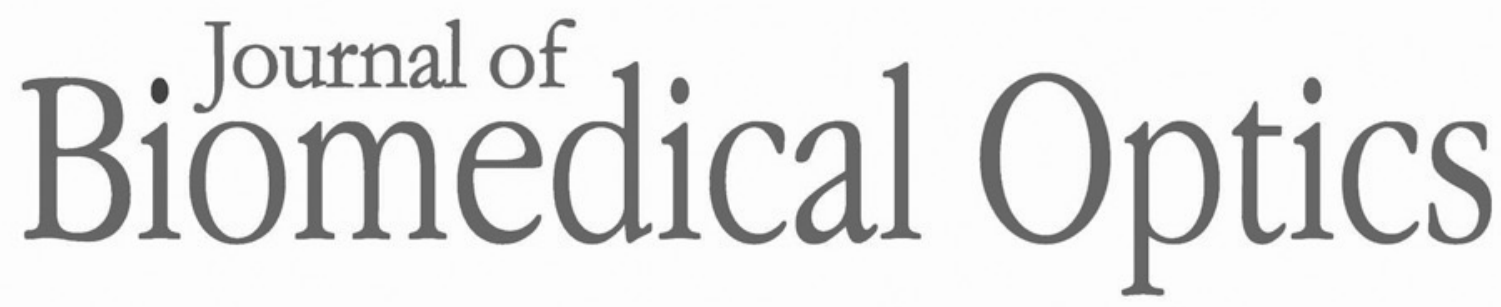

BiomedicalOptics.SPIEDigitalLibrary.org

\title{
JBO 2015 List of Reviewers
}

\section{SPIE.}


The Journal of Biomedical Optics would like to sincerely thank the following individuals who served as reviewers in 2015 . The success of our publication hinges on the voluntary contributions of time and energy put forth by these professionals.

\begin{tabular}{|c|c|}
\hline Ibrahim Abdulhalim & Bernhard Baumann \\
\hline Thomas Abraham & Pinky Bautista \\
\hline Nils Afseth & Martin Becker \\
\hline Ali Afshari & Artur Bednarkiewicz \\
\hline Anant Agrawal & Andrei V. Belikov \\
\hline Guillermo Aguilar & Muyinatu Bell \\
\hline Aaron Aguirre & Tos TJM Berendschot \\
\hline Balpreet Ahluwalia & Thomas Berer \\
\hline Peter Ahnelt & Andrew Berger \\
\hline Flavio Aimbire & Kathrin Berkner \\
\hline Kathleen Alam & Peter Berlien \\
\hline Jarmo Alander & Edouard Berrocal \\
\hline Erik Alerstam & Xiaohong Bi \\
\hline Sergey Alexandrov & Liheng Bian \\
\hline Robert Alfano & Vasudevan Biju \\
\hline Thomas Allen & Philip Bingham \\
\hline Umar Alqasemi & Tiziano Binzoni \\
\hline Clemens Alt & Alexandru Biris \\
\hline Arun Anand & John Birk \\
\hline Afrouz Anderson & Roger Bisby \\
\hline Pamela Anderson & John Bischof \\
\hline Masatoshi Ando & Nrusingh Biswal \\
\hline Cintia Andrade & Albert Claude Boccara \\
\hline Maciej Antkowiak & Thomas Bocklitz \\
\hline Jean-Pierre Antoine & Martin Booth \\
\hline Kazuhiro Aoki & Stephen Boppart \\
\hline Lorilee Arakaki & Ekaterina Borisova \\
\hline Praveen Arany & Richard Bouchard \\
\hline Samuel Arba Mosquera & Brett E. Bouma \\
\hline M. Hassan Arbab & Stephen G. Bown \\
\hline Yasaman Ardeshirpour & Boy Braaf \\
\hline Alawiah Ariffin & Roberto Braga Jr. \\
\hline Justo Arines & John Briers \\
\hline John W. Arkwright & Ralf Brinkmann \\
\hline Cosimo Arnesano & J. Quincy Brown \\
\hline William Ash III & Nico Bruining \\
\hline Kavita Aswani & Joanna Brunker \\
\hline Joonas Autio & Erin Buckley \\
\hline Mohammad Avanaki & Juan Bueno \\
\hline Markus Axer & Lisa Buergermeister \\
\hline Hasan Ayaz & Peter Burgholzer \\
\hline Saulius Bagdonas & Miran Burmen \\
\hline Hua Bai & Andrew Burnett \\
\hline Yuqiang Bai & Alexander Bykov \\
\hline Yuquiang Bai & Murat Canpolat \\
\hline Turki Bakhsh & Stefan Carp \\
\hline Madhusudhanan Balasubramanian & Maria Cekanova \\
\hline Michela Balconi & Mehmet Celenk \\
\hline Lucia Ballerini & Jonathan Celli \\
\hline Duoduo Bao & Judit Chamorro-Servent \\
\hline Timothy Baran & James Chan \\
\hline Randall Barbour & Nick Chandler \\
\hline Margarida Barroso & Rajesh Chandramohanadas \\
\hline Thomas Barstow & Lingqian Chang \\
\hline Randy Bartels & Wei-hau Chang \\
\hline Jennifer Barton & Rajeev Chaudhary \\
\hline Virginijus Barzda & Chih-Hsin Chen \\
\hline Alexey Bashkatov & Hsi-Chao Chen \\
\hline
\end{tabular}

Ibrahim Abdulhalim

Anant Agrawal

Guillermo Aguilar

Aaron Aguirre

Balpreet Ahluwalia

Jarmo Alander

Sergey Alexandrov

Umar Alqasem

Afrouz Anderson

Maciej Antkowiak

Samuel Arba Mosquera

Alawiah Ariffin

Mvanaki

Saulius Bagdonas

Turki Bakhsh

Michela Balconi

Lucia Ballerini

Margarida Barroso

Thomas Barstow

Alexey Bashkatov
010102-1

\author{
Nanguang Chen \\ Wei Chen \\ Wen Chen \\ Xu Chen \\ Xueli Chen \\ Yu Chen \\ Yu Chen \\ Zhenqiang Chen \\ Ji-Xin Cheng \\ Ran Cheng \\ Olga Cherkasova \\ Dan Chicea \\ Sangyeon Cho \\ Regine Choe \\ Bernard Choi \\ Wonshik Choi \\ Michael Choma \\ Xinyuan Chong \\ Kengyeh "Ken" Chu \\ Shi-Wei Chu \\ Riccardo Cicchi \\ Marcus Cicerone \\ Inci Cilesiz \\ Ludmila Otilia Cinteza \\ Neil Clancy \\ Ela Claridge \\ Eric Clarkson \\ Davide Contini \\ Julia Craven-Jones \\ Simona Cristescu \\ Giovanni Crosta \\ John Crowe \\ Maria Luisa Cruz Lopez \\ Brian Culshaw \\ Andrea Curatolo \\ Anabela Da Silva \\ Gislin Dagnelie \\ Eugenie Dalimier \\ Simon Danby \\ Marcos Dantus \\ Arun Darbar \\ Vincent Ricardo Daria \\ Cynthia Darling \\ Maxim Darvin \\ Raktim Dasgupta \\ Anjul Davis \\ Scott C. Davis \\ Daniel de Bruin \\ Elbert de Josselin de Jong \\ Xose Luis Dean Ben \\ Stanislas Deby \\ Hamid Dehghani \\ Michael DellaVecchia \\ Francois Delori \\ Stavros G. Demos \\ Bin Deng \\ Shengling Deng \\ Eustace L. Dereniak
}


Parmeswaran Diagaradjane

Gerald J. Diebold

Charles DiMarzio

Zhihua Ding

U. S. Dinish

Mamadou Diop

Ayca Dogan Mollaoglu

Simon Doran

Alexander Doronin

Tatjana Dostalova

Alexandre Douplik

Bruce Drum

Dane Drutis

Lian Duan

Arnaud Dubois

Alexander Dubolazov

Alfredo Dubra

Matthieu Dubreuil

Michel A. Duguay

Austin Duke

Dan Dumitras

Graham Dunn

Christopher Dunsby

Turgut Durduran

Glenn Edwards

Adam Eggebrecht

Martin Ehler

Ahmed El Kharbotly

Audrey Ellerbee

Jonathan Elliott

Daniel Elson

Tawfig Eltaif

Valentina Emiliani

Annika Enejder

Thomas Ertl

Galileo Escobedo

Nicholas Everdell

Avishay Eyal

Raul Fainchtein

Qiyin Fang

Ye Fang

Felix Fanjul-Velez

Sergio Fantini

Peter Farnbach

Ivan Fedosov

Baowei Fei

Felix Feldchtein

Adolf Fercher

Farzad Fereidouni

Enrique-Josua Fernandez

Raffaele Ferri

Wolfgang Fink

Dror Fixler

Reginald Fletcher

Matthew Ford

Thomas Foster

Seyyed Hamed Fouladi

John Fourkas

Eduardo Fregnani

Matthias Frentzen
Daniel Fried

Nathaniel Fried

Ling $\mathrm{Fu}$

Katsumasa Fujita

Shinichi Fukuda

John Fumo

W. Robert J. Funnell

Ekaterina Galanzha

Thilo Gambichler

Ute Gamm

Angarai Ganesan

Israel Gannot

Feng Gao

Hao Gao

Liang Gao

Simon Gao

Wanrong Gao

Alejandro Garcia-Uribe

Craig Gardner

Jiajia $\mathrm{Ge}$

Grigory Gelikonov

Werner Gellermann

Luc Gendre

Elina Genina

Thomas Gensch

Csilla Gergely

Bernard S. Gerstman

Ara Ghazaryan

Nirmalya Ghosh

Paolo Giacometti

Summer Gibbs

Adam Gibson

Jose Gil

Fiona Gilbert

Sylvain Gioux

John Girkin

Pinhas Girshovitz

Adam Glaser

Randolph Glickman

Tatiana Globus

Arthur Gmitro

Anuradha Godavarty

Bo Gong

Iwona Gorczynska

Dimitris Gorpas

Francois Goudail

Kathleen Gough

Andris Grabovskis

Bruno Grassi

Karl Greulich

Gurtej Grewal

Ireneusz Grulkowski

Min Gu

Yueqing $\mathrm{Gu}$

Fada Guan

Rui Guerra

James Guggenheim

Anthony Gummer

Han-Wen Guo

Huanqing Guo
Tuan Guo

Zijian Guo

Gunnsteinn Hall

Markus Haltmeier

Takafumi Hamaoka

Michael Hamblin

Billy Hammond

Jae-Ho Han

Xiaoxing Han

Steen Hanson

Anderson Hara

David Harris

Andrew R. Harvey

Tayyaba Hasan

Moinuddin Hassan

Carole Hayakawa

Joseph Hayward

Jeremy Hebden

Rodney Heckaman

Martin Hedegaard

H. Michael Heise

Ricky Hennessy

Boris Hermann

Kevin Hewitt

Raimund Hibst

Ho-pui (Aaron) Ho

Alison Hobro

Roman Hochuli

Ansgar Hohmann

Gary Holtom

Jennifer Holtzman

Timothy Holzheimer

Jisoo Hong

Yoko Hoshi

Nicolette Houreld

Vladimir Hovhannisyan

Yi-Sing Hsiao

Jiming $\mathrm{Hu}$

Sijung $\mathrm{Hu}$

Chih-Hsien Huang

Jinxin Huang

Jiwei Huang

Yong Huang

Zheng Huang

Ramon Huerta

Gereon Huettmann

Michael Hughes

Anne Humeau-Heurtier

Jennifer Hunter

Theodore Huppert

Mark Hutchinson

M. Shane Hutson

K.C. Hwang

Emmett lentilucci

Takeshi Imamura

Martin Isabelle

D. Robert Iskander

Andrei Ivanov

Robert Ivkov

Mohan Jacob 
Steven Jacques

Volker Jaedicke

Michael Jaeger

Jared Jagdeo

Dainis Jakovels

Hyounguk Jang

Jaeduck Jang

Nicolas Javahiraly

Pierre Jeannesson

Michael Jenkins

Michael Jermyn

Minbiao Ji

Dagong Jia

Yali Jia

Huabei Jiang

Shudong Jiang

Shuliang Jiao

Jose Ramon Jimenez Cuesta

Javier Jo

Thomas Joergensen

Robert Jones

Cecil Joseph

Bodh Jugdutt

Young-wook Jun

Byungjo Jung

Jae-Hyun Jung

Asta Juzeniene

Jana Kainerstorfer

Samata Kakkad

Vyacheslav Kalchenko

Jeroen Kalkman

Guido Kamphuis

Alexei Kamshilin

Katsuhiro Kanamori

DongKyun Kang

Pilgyu Kang

Stephen Kanick

Dimitrios Kapsokalyvas

Tiina Karu

Amir H Kashani

Rachel Kast

Yoshimasa Kawata

Bjoern Kemper

Brendan Kennedy

David Kessel

Morteza Khaleghi

Alexander Khmaladze

Davood Khodadad

Mohammad Khosroshahi

Alwin Kienle

Masashi Kiguchi

Anthony Killard

Arnold Kim

ChangSeok Kim

Chulhong Kim

Do-Hyun Kim

Jae Kim

Jong-Ki Kim

Myun Sik Kim

Nam Keun Kim
Seung-Woo Kim

Young-jin Kim

Youngchan Kim

Akatsuki Kimura

P. Ewen King-Smith

Matti Kinnunen

Mikhail Kirillin

Sean J. Kirkpatrick

Toshihiko Kiwa

Roman Kleindienst

Aleksandra Klimas

George Knopf

Alex Ko

Irene Kochevar

Eva Kocisova

Shunsaku Koga

Won-Gun Koh

Matthias Kohl-Bareis

Satoru Kohno

Nikiforos 'Nik' Kollias

Lingbo Kong

George Koob

Vesa Korhonen

Kivanc Kose

Nalinikanth Kotagiri

Sri-Rajasekhar Kothapalli

F Kozel

Vitaly Krasovskii

Emilie Krite Svanberg

Silke Krol

Wanne Kromdijk

Alexander Krueger

Joerg Krueger

Andrey Kruglov

Wolfgang Kuebler

Abhishek Kumar

Sanjay Kumar

Vikas Kundra

Leionid Kunyansky

Roman Kuranov

Juergen Lademann

Puxiang Lai

Stephen Lam

Guy Lamouche

Birgit Lange

Kirill Larin

Bjorg Larson

Bahman Lashkari

Jan Laufer

Julien Laverdant

Xavier Le Guevel

Silas Leavesley

Byeong Ha Lee

Byoungho Lee

Changho Lee

Chau-Hwang Lee

Daniel J Lee

Hsiang-Chieh Lee

Kisung Lee

Sang-won Lee
SanYun Lee

Tim Lee

Kevin Lehmann

Catia Leitao

Denis Leroux

Paul LeVan

Richard Levenson

Zachary Levine

David Levitz

Changqing $\mathrm{Li}$

Chunhui Li

Guo Li

$\mathrm{HaO} \mathrm{Li}$

Jiasong $\mathrm{Li}$

Lei Li

Lin Li

Meng-Lin Li

Nan Li

Qinggele Li

Qingli Li

Rui Li

Shiping $\mathrm{Li}$

Ting $\mathrm{Li}$

Kaicheng Liang

Rongguang Liang

Martin Liess

Lothar Lilge

Magnus Lilledahl

Jin-Ik Lim

Charles Lin

Li Lin

Sung-Jan Lin

Wei-Chiang Lin

Norbert Linz

Norman Lippok

Tom Lister

Cheng Liu

Gangjun Liu

Hanli Liu

Jun Liu

Quan Liu

Tzu-Ming Liu

Wenzhong Liu

Xuan Liu

Xuan Liu

Yang Liu

Yang Liu

Yuan Liu

Stephen Lockett

Noemi Lois

Yang Lou

Gordon Love

Pablo Loza-Alvarez

Fake Lu

Zenghai Lu

Holger Lubatschowski

Gerald Lucassen

Brian Lund

David Lund

Jennifer Lynch 
Hui Ma

Li Ma

Mark Mackanos

Boris Majaron

Hassaan Majeed

Angshul Majumdar

Atsushi Maki

Bilal Malik

Zvi Malik

Scott Manalis

Andreas Mandelis

Thomas Mang

Srirang Manohar

Marija Marcan

Alzbeta Marcek Chorvatova

Zbignevs Marcinkevics

Laura Marcu

Thibault Marin

Fabrizio Martelli

Laura Martinez Maestro

Herculano Martinho

David Mas

michiyuki matsuda

Mami Matsukawa

Kenta Matsumura

Lev Matveev

Amaan Mazhar

David McClatchy III

Gail McConnell

Kevin McCully

James Mcgrath

Andy McNaught

Mike McShane

Patrick McVeigh

Igor Medintz

Alena Medrado

Igor Meglinski

Martina Meinke

Joerg Meister

Arcangelo Merla

Jerome Mertz

Michel Meunier

Matija Milanic

Thomas Milner

Sung-Wook Min

Atsushi Mine

Mustafa Mir

Julian Moger

Serge Monneret

Inkyu Moon

Michael Morris

Claudia Mota

Judith Mourant

Maya Mueller

Mircea Mujat

Timothy Muldoon

Peter Munro

Kartikeya Murari

Christine Murphy

Ian J. Murray
Vadakke Mathan Murukeshan

Nikhil Mutyal

Oleg Nadiarnykh

Annemarie Nadort

Viktor Nadtochenko

Eman Namati

Otilia Nasui

Maria Navas-Moreno

Marcella Nebbioso

Seo-Mie F. Nee

Praveen Nekkar Rao

Eddie $\mathrm{Ng}$

Thu-Mai Nguyen

Brandon Nichols

Dimitrios Nikolelis

Meir Nitzan

Farouk Nouizi

Tatiana Novikova

Robert Nuster

Dennis O'Neal

Thomas O'Sullivan

John Oghalai

Seung Jae Oh

Seungeun Oh

Yoshihiro Ohmiya

Eiji Okada

Amy Oldenburg

Nancy Oleinick

Luis Oliveira

Jeffrey Oliver

Sylwia Olsztynska-Janus

Xavier Orlik

Sergei Orlov

Pal Ormos

Noe Ortega-Quijano

Cees Otto

Yukihiro Ozaki

Cynthia Pagba

Gregory Palmer

Guenther Paltauf

Yingtian Pan

Rishikesh Pandey

Shuo Pang

Nicolai Panikov

Asael Papour

Sapun Parekh

Nivaldo Parizotto

B. Hyle Park

HyunJoo Park

Jae-Hyeung Park

YongKeun Park

Ashwin Parthasarathy

Chetan Patil

Chetan Patil

Michael Patterson

Michal Pawlowski

George Peavy

Giancarlo Pedrini

Ivan Pelivanov

Katherine Perdue
T. Pfefer

Kevin Phillips

Daqing Piao

Mark C. Pierce

Antonio Luiz Pinheiro

Michael Pircher

Kristjan Plaetzer

Sergey Plotnikov

Jerzy Plucinski

David Plusquellic

Gretel Png

Brian Pogue

Ting-Chung Poon

Alexey Popov

Rachela Popovtzer

Juergen Popp

Eric Potma

Samuel Powell

Vivek Prabhu

Asima Pradhan

Jaya Prakash

Manojit Pramanik

Yang $\mathrm{Pu}$

Haridas Pudavar

Xulei Qin

Jianjun Qiu

Zhen Qiu

Junle Qu

Bernard Querleux

Kyle Quinn

Harsha Radhakrishnan

Milind Rajadhyaksha

Narasimhan Rajaram

Sripriya Ramamoorthy

Jessica Ramella-Roman

Abel Ramoelo

Venkat Ramshesh

Janaka Ranasinghesagara

Bin Rao

Aniruddha Ray

Daniel Razansky

Scott Read

Peter Rechmann

Caitlin Regan

Jean Rehbinder

Fred Reinholz

Karen Reiser

Hongwu Ren

Kui Ren

Jungtae Rha

Shachar Richter

Monika Ritsch-Marte

William Roberts

Francisco Robles

Lidiany Rodrigues

Leonardo Roever

Jeremy Rogers

Kelly Rogers

Olaf Rolinski

Jannick Rolland 
Andrea Romani Jessica Rosenholm John J. Rosowski Gerry Ross Alexander Rubenchik Angelika Rueck Phaltane Ruhi Pablo Ruiz David Russell Christopher Rylander H. Grady Rylander III Rolf Saager Caetano Sabino

Alireza Sadr Partha Sahu Sava Sakadzic Hamideh Salehi Hassan Salehi Ahmad Salman Christopher Salthouse Ota Samek Kimberley Samkoe Ylenia Santoro Ramesh Sarangapani Mohammad Sarshar Marinko Sarunic Angelo Sassaroli Hidetoshi Sato Chikara Satoh Jon Scaffidi Giuliano Scarcelli Leopold Schmetterer Michael Schmitt Tilman Schmoll Joshua Schoenly Felix Scholkmann Karl Schulmeister James Schweigerling Dietrich Schweitzer David Schwenninger Henrik Schytz

Wolf Seka

Oxana Semyachkina-Glushkovskaya

Carmen Serrano

Alexey Seteikin

Eva Sevick-Muraca

Kristian Sexton

Natan Shaked

Yu Shang

R. Anthony Shaw

Ryan Shelton

Jin Shen

Yihui Shen

Lingyan Shi

Wei-Chuan Shih

Sehyun Shin

Dustin Shipp

Dustin Shipp Jeffrey W. Shupp M. Shyu
Garth Simpson

Srikanth Singamaneni

Rakesh Singh

Laura Sironi

Melissa Skala

Alex Small

Zachary Smith

Emil Sobol

Stavroula Sofou

Young-Soo Sohn

Steven Solga

Gabor Somfai

Chul Gyu Song

Liang Song

Xiaolei Song

Laura Sordillo

Brian S. Sorg

Janis Spigulis

Robert Splinter

Thomas Allen Springer

Tomasz Spyt

Vivek Srinivasan

Sachin Srivastava

Ronald Sroka

Keith St. Lawrence

Georgios Stamatas

Wiendelt Steenbergen

Herbert Stepp

David Stork

Chiara Stringari

Tomas Stromberg

Mathias Strupler

Bruce Stuck

lain Styles

Hiroo Suami

Hrebesh Subhash

Klaus Suhling

Ebraheem Sultan

Carry Sun

Chi-Kuang Sun

Chia-Wei Sun

Ching-Cherng Sun

Ulas Sunar

Yongjin Sung

Lars Svaasand

Zdenek Svindrych

Mahesh Swami

Johannes Swartling

Daniel Szlag

Henryk Szmacinski

Hasim Ozgur Tabakoglu

Ilias Tachtsidis

Pinyo Taeprasartsit

Allen Taflove

Enrique Tajahuerce

Toshiki Tajima

Setsuo Takatani

Jianbo Tang

Rui Tang

XiaoHong Tang
Yuankai Tao

Paola Taroni

Tanja Tarvainen

Darrell Tata

Joel Teichman

James Terner

Gregor Tevz

Nitish Thakor

Lotte Themstrup

Suresh Thennadil

Robert Thomas

Alexander Thompson

Oliver Thompson

Richard Thompson

Krishna Thyagarajan

Francois Tiaho

Jian Tian

Jie Tian

Lei Tian

Karissa Tilbury

Tomasz Tkaczyk

Shanbao Tong

Kimani Toussaint

Jens Traeger

Valentina Trapani

Meng-Tsan Tsai

Sheng-Hao Tseng

Kevin K. Tsia

Andrew Tsourkas

Vassiliy Tsytsarev

Jean-Michel Tualle

Valery Tuchin

James Tunnell

Sylvia Turrell

Aisada Uchugonova

Giovanni Ughi

Fasee Ullah

Hafeez Ullah

Paul Urayama

Urs Utzinger

Benjamin Vakoc

Niksa Valim

Hubert van den Bergh

Sabine van Huffel

Fijs W. B. van Leeuwen

Gijs van Soest

Martin vandeVen

Gracie Vargas

Darko Vasiljevic

Mathieu Vedel

Andreas Velten

Rudolf Verdaasdonk

Wim Verkruysse

John Viator

Karthik Vishwanath

I. Alex Vitkin

Vadim Viviani

Alfred Vogel

Steven Vogel

William Vogt 
Andreas Volkmer

Marc Vuylsteke

Laura Waller

Laurence Walsh

Ge Wang

Hao Wang

Hequn Wang

Hongkai Wang

Hui Wang

Jianting Wang

Kun Wang

Lidai Wang

Pu Wang

Shang Wang

Tianheng Wang

Tianyi Wang

Wei Wang

Xinheng Wang

Xueding Wang

Zhao Wang

Zheng Wang

Neville Washburn

Michiko Watanabe

Naoki Watanabe

Adam Wax

Chenwei Wei

Qingshan Wei

Steven Wei

Wei Wei

Xunbin Wei

Nicolas Weiss

Wendy Wells

David Wertheim

H.T. Whelan

Harvey Wigdor

Martin Wolf

Ursula Wolf

Brian Wong

Kenneth Kin-Yip Wong

Huawen Wu
Jigang Wu

Yicong Wu

Lei $\mathrm{Xi}$

Jun Xia

Wenfeng Xia

Han Xiao

Tuqiang Xie

Chen Xu

Chris Xu

Min Xu

Ronald Xu

Xiangqun $\mathrm{Xu}$

Ping Xue

Vladislav Yakovlev

Yukio Yamada

Masahiro Yamanari

Bin Yang

Huihua Yang

K. L. Yang

Zhengyi Yang

Gang Yao

Junjie Yao

Xincheng Yao

Mohammad Yaseen

Jing Yong Ye

Yin Yeh

Dvir Yelin

Xin Yin

Christopher Yip

Elizabeth Yoder

Arjun Yodh

Bin Yu

Guoqiang Yu

Xiao Yu

Baohong Yuan

Zhen Yuan

Meryem Yucel

Dmitry Yudovsky

Maxim Yurkin

Giovanni Zaccanti
Vladimir Zaitsev

Pavel Zakharov

Christian Zakian

Zeev Zalevsky

Jihad Zallat

Livia Zarnescu

Robert Zawadzki

Dejan Zecevic

Haishan Zeng

Myriam Zerrad

Anqi Zhang

Hao Zhang

Jun Zhang

Kang Zhang

Limin Zhang

Rongxiao Zhang

Xiaofeng (Steve) Zhang

Xu Zhang

Xu Zhang

Jianhua Zhao

Jie Zhao

Mei-Xia Zhao

Qing Zhao

Yan Zhao

Guoan Zheng

Evgeny Zherebtsov

Chao Zhou

Qifa Zhou

Wenjing Zhou

Yong Zhou

Caigang Zhu

Chao-Zhe Zhu

Dan Zhu

Quing Zhu

Timothy C. Zhu

Yizheng Zhu

Daniel Zicha

Andres F. Zuluaga

Chao Zuo

Andrei Zvyagin 